Classification

Physics Abstracts

$6470-61.12-6140$

\title{
Bilayers Versus Micelles in Very Dilute Surfactant Ternary Systems
}

\author{
M. Filali, J. Appell and G. Porte \\ Groupe de Dynamıque des Phases Condensées $\left({ }^{*}\right)$, Case 026, Unıversıté Montpellier II, \\ 34095 Montpellier Cedex 05, France
}

(Received 19 December 1994, revised 25 February 1995, accepted 16 March 1995)

\begin{abstract}
Using neutron scattering, we investigate the structure at local scale of very dilute solutions of a quasiternary surfactant system. For such systems consisting of surfactant and cosurfactant in brine, a critical line is often found in the very dilute range of the phase diagram where the L3 sponge domain is continuously connected to the micellar domain Our purpose is to examine the evolution of the morphology of the surfactant aggregates close to this critical line Starting in the L3 sponge domain and approaching the transition line, we find that the neutron scattering patterns deviate more and more from that of an infinite bilayer Futhermore, far away on the other side of the critical line, the neutron scattering patterns reveal that the intial bilayers have been replaced by long $1 \mathrm{D}$ cylindrical micelles. These findings are discussed in terms of the two different mechanisms that have been considered in the literature as being possibly at the origin of the critical behaviour the symmetry breaking of the sponge and the spontaneous tearing of the bilayer.
\end{abstract}

\section{Introduction}

The structure and the physical properties of the L3 (sponge) phase of surfactant systems have been studied extensively [1-4] and they are presently reasonably well-understood. This phase is observed in conditions (temperature, salinity, and cosurfactant-to-surfactant ratio in the case of a mixed system) such that the surfactant molecules prefer to self-assemble into 2D-bilayers. But in contrast with the usual lamellar phase, it shows no long-range order. The infinite bilayer is arranged and bent in space so as to be multiconnected to itself along all directions over macroscopic distances. The phase is in general stable all over the moderate dilution range (i.e., typically in the 1 to $20-30 \%$ volume fraction range) just like the lamellar phase which is usually observed close to L3 in the phase diagram. At high dilution, one encounters different situations depending of the systems investigated. In some cases, the L3 structure simply phase separates at some stage with a very dilute L1 dispersion of micelles. For some other systems [5], the L3 domain is continuously connected to the micellar L1 domain at high dilution.

$\left({ }^{*}\right)$ UA 233 du CNRS

(C) Les Editions de Physique 1995 
This means that, starting from the infinite multiconnected membrane in L3, it is possible to move in the phase diagram and reach a random dispersion of micelles continuously with no macroscopic phase separation. Two scenarios have been considered in the literature to describe this experimental fact consistently. The first is based on theoretical considerations about the symmetry of the L3 phase. At moderate concentration, the membrane in L3 separates the space in two statistically equivalent subvolumes: this statistical symmetry of the phase simply reflects the intrinsic local symmetry of the membrane with respect to its mid surface. At high dilution, however, the global symmetry could well break spontaneously. Below the corresponding critical concentration, the membrane disconnects progressively, finally ending up in the form of a random dispersion of vesicles and/or micelles. The consequences of this notion of a hidden symmetry in the L 3 sponge phase, as well as those of the possible symmetry breaking, have been examined in great detail both theoretically and experimentally for the last few years $[3,5-7]$.

The other scenario, introduced by Huse and Leibler [8], was motivated by considerations on the statistical distribution of defects, such as "holes", that might form spontaneously on the bilayer: since the free energy of a hole of finite size is certainly finite, its probability of formation is not zero. More precisely, the excess free energy involved in forming holes can be characterised by a line tension $\lambda$ (i.e., the free energy per unit length of an edge forced on the membrane). At high $\lambda$, holes are scarce and small and the description of the L3 structure in terms of an infinite bilayer multiconnected over macroscopic distances indeed survives. At low enough $\lambda$, however, the number density and the average size of the holes increase so much that the infinite bilayer is finally torn into a dispersion of finite disconnected pieces. An important prediction of reference [8] is that, before this happens, there exists a "critical" value $\lambda_{c}$ of $\lambda$ where the average contour length of the edge loops diverges. The membrane at $\lambda_{\mathrm{c}}$ is still infinite but bears infinite edges: this special structure was called "the sponge with free edges" by Huse and Leibler. Therefore, just like the above-described symmetry breaking, the spontaneous tearing of the membrane must be considered as a critical phenomenon.

In reference [9], we investigated in detail the L3-phase behaviour of the OBS system (Naoctylbenzene sulfonate) / $\mathrm{n}$-pentanol / brine $(0.5 \mathrm{M} \mathrm{NaCl})$ in the very dilute range. As shown in Figure 1, the phase diagram of this system shows a continuous path connecting at high dilution the domain of stability of L3 to that of the L1 micellar phase. The dotted line shown in Figure 1 into the isotropic one phase domain, represents the line where the intensity of the scattered light shows a sharp maximum suggesting there a critical behaviour (called hereafter the line of maximum turbidity: LMT). Indeed, this phase diagram with the LMT has a geometry very similar to that of the formerly mentioned system investigated of references [5,7]. However, while the authors in references $[5,7]$ mainly focused their experimental study on the vicinity of the coexistence line of their system, we studied more particularly the LMT far from the coexistence line deep inside the one phase isotropic domain: i.e., along a path denoted l.s. in Figure 1, at constant total surfactant + cosurfactant concentration, changing only the cosurfactant-tosurfactant ratio. Several features of the light scattered along such paths were found inconsistent with the picture where the LMT is the line where the symmetry breaking takes place. So, the possibility was considered in reference [9] that the critical behaviour observed at the LMT could rather be related to the spontaneous tearing of the membrane described just above according to the Huse and Leibler scenario.

It is important in this context to check the presence of structural defects, such as edges, in the vicinity of the LMT. The technique (light scattering) used in [9] probes the sample at large scale (low wave vector $\mathbf{q}$ ). It only provides information on the concentration fluctuations and gives no insight on what happens at local scale at the transition line. Our purpose here is to complement our former light scattering study of the critical behaviour at the LMT and 
to collect structural data at smaller scale length: we use neutron scattering and try to observe the morphological transformations of the membrane when moving in the phase diagram from one side of the LMT to the other.

\section{Experiments}

Neutron scattering has often been used in the field of amphiphilic systems in order to determine the size and shape of primary surfactant structures. In particular, the morphology sequence that takes place in ternary systems at moderate amphiphile concentration, when increasing the alcohol-to-surfactant ratio (globules, long cylinders in the isotropic L1 phase, bilayers in the smectic lamellar phase and in the isotropic L3 phase), was actually demonstrated by this technique [1]. Indeed the situation is specially simple when the interactions between the objects can be neglected: the neutron scattering pattern is then dominated by the form factor. This is the case here since we deal with very dilute samples in presence of high $\mathrm{NaCl}$ concentrations.

However, a specific difficulty with very dilute samples arises due to the finite solubility of the amphiphiles. When changing the pentanol-to-OBS ratio at constant total amphiphile concentration (such as in the path l.s in Fig. 1), we do not know the respective proportion of amphiphiles which self assemble into micellar objects or bilayers and that which remain molecularly dispersed in the aqueous solvent (note that the solubility of pentanol in brine at $20{ }^{\circ} \mathrm{C}$ is of the order of $5 \%$ ). This makes it difficult to compare quantitatively the scattering patterns obtained from different samples. To avoid this problem, we use here the salinity as the monitoring parameter rather than the pentanol to OBS ratio. As shown in reference [9], essentially identical light scattering behaviour is observed at the LMT no matter whether we use the salinity rather than the alcohol to surfactant ratio as the control parameter. So we start at a composition on the L3 side of the LMT ( $\mathrm{P}_{1}$ in Fig. 1), we keep both the pentanol and the OBS concentrations constant $\left(C_{\text {pentanol }}=0.0188 \mathrm{~g} / \mathrm{g}\right.$ and $\left.C_{\text {OBS }}=0.0062 \mathrm{~g} / \mathrm{g}\right)$ and move through the critical conditions by decreasing the salinity of the solvent only. As long as the salinity range used is not too broad, it should not affect the cmc of the OBS and the solubility of the pentanol too much, so that we can safely assume that the relative quantity of

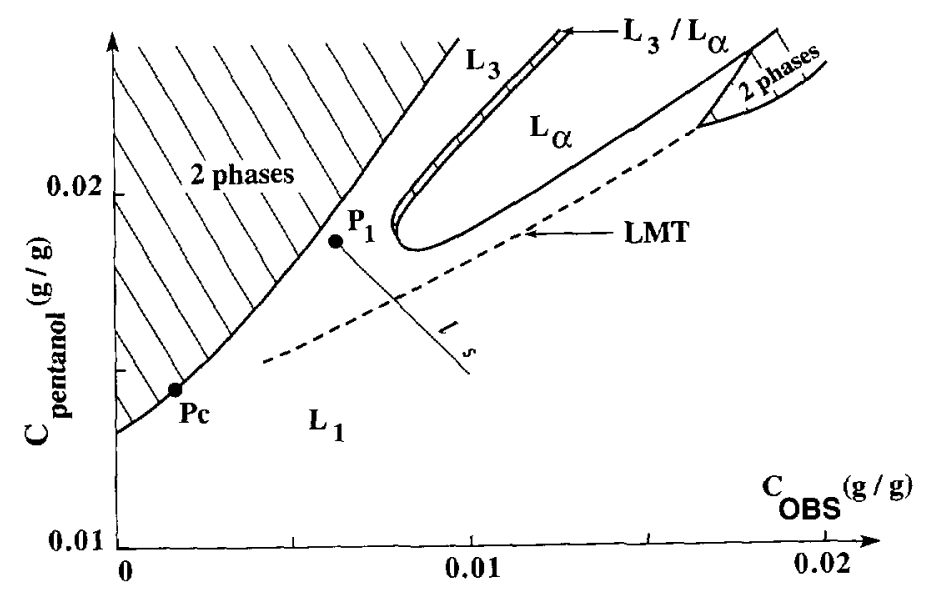

Fig. 1. - Very dilute part of the phase diagram of the system OBS / n-pentanol / brine (0.5 M NaCl). The dotted line is the LMT where the scattered light intensity seems singular. Point $P_{1}$ represents the composition in amphiphles for the samples investigated in this study. 


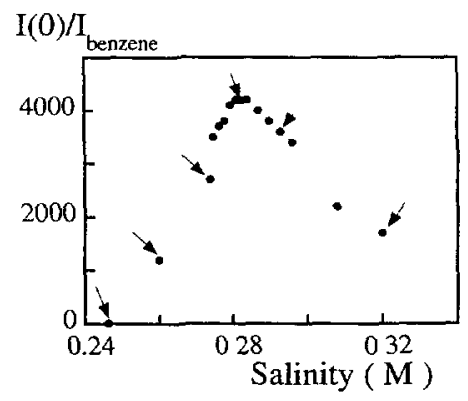

Fig. 2. - Intensity of the light scattered at zero angle as a function of the salınity in $\mathrm{D}_{2} \mathrm{O}$ solutions. All samples have the same composition in amphiphles $\left(C_{\mathrm{OBS}}=062 \times 10^{-2} \mathrm{~g} / \mathrm{g}\right.$ and $C_{\text {pentanol }}=$ $1.88 \times 10^{-2} \mathrm{~g} / \mathrm{g}$ corresponding to point $\mathrm{P}_{1}$ in Fig. 1) but different salinities The arrows correspond to the samples studied by neutron scattering.

amphiphiles in the self-assembled state is constant from one sample to the other.

For contrast reasons, $\mathrm{D}_{2} \mathrm{O}$ has to be used rather than $\mathrm{H}_{2} \mathrm{O}$. We have measured (Fig. 2) the intensity of the light scattered by $\mathrm{D}_{2} \mathrm{O}$ samples having the above-mentioned pentanol and OBS compositions as a function of the salinity of the brine. Having so determined the critical salinity in $\mathrm{D}_{2} \mathrm{O}$, we have selected six samples for neutron scattering measurements. These samples are denoted by arrows in Figure 2 and their respective salinities given in the captions of Figures 3-5. The neutron scattering measurements are performed on the line PACE at the Léon Brillouin Laboratory (Saclay, France), using standard experimental procedure and the data treatment. In order to scan a sufficiently large $q$-range, different neutron wavelengths (6 and $10 \AA$ ) and different sample-to-detector distances (1.5 and $5 \mathrm{~m}$ ) have been used: so $q_{\mathrm{min}}=5 \times 10^{-3} \AA^{-1}$ and $q_{\max }=0.22 \AA^{-1}$. Note that for the amphiphile concentration chosen here, the "cell" size expected for the symmetric sponge is of the order of $1500 \AA$; so all data are taken here at wave vectors larger than that of the sponge correlation maximum $\left(q_{\mathrm{m}}=2 \pi / 1500=4 \times 10^{-3} \AA^{-1}\right)$. They only reflect the morphology at local scale in the samples. Since all samples have presumably identical concentrations of amphiphiles in the self-assembled state, we compare their respective scattering patterns directly with no requirement for any intensity rescaling prefactor. The resulting scattering patterns are presented in Figures 3-5.

Let us first consıder the pattern of the sample with the highest salinity. From its position relative to the critical salinity, we expect it to correspond to the regular L3 sponge phase. The scattering pattern should therefore be simply identical to that of a random dispersion of very large defect free $2 \mathrm{D}$ bilayer over the entire $q$-range investigated here:

$$
I(q) \sim q^{-2} \exp \left(-\delta^{2} q^{2} / 12\right)
$$

The scattering is hence shown in Figure 3a using the representation appropriate for 2D objects, i.e., $\ln \left(q^{2} I(q)\right)$ versus $q^{2}$. As expected, a straight line is obtained at low $q$, the negative slope of which is, in principle, indicative of the finite thickness $\delta$ of the bilayer (slope $=-\delta^{2} / 12$ ). Thus we obtain $\delta=15 \AA$. Note that, since the contrast mainly arises from the protonated amphiphiles versus the heavy water solvent, $\delta$ essentially represents the thickness of the aliphatic core of the bilayer. In this context, its value is in good agreement with the estimate of the total thickness of the OBS-pentanol bilayer ( $20 \AA$ ) obtained by another method in reference [10]. For the sake of comparison, we also show in Figure $3 b$ the scattering pattern of the same sample using the representation appropriate for $1 \mathrm{D}$ cylindrical objects, i.e., $\ln (q I(q))$ versus $q^{2}$. Indeed in this 

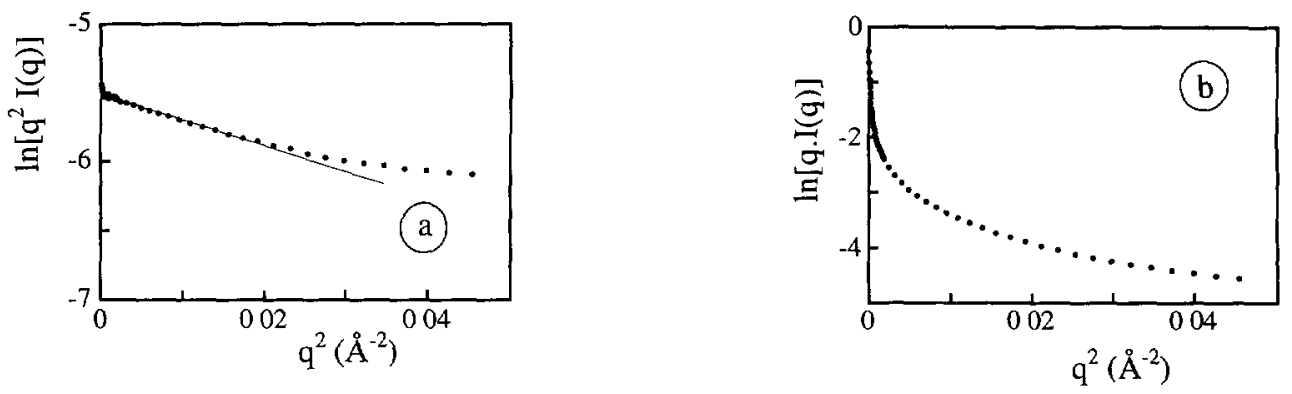

Fig. 3. - a) Neutron scattering pattern of the sample with salinity $0320 \mathrm{M}$ in the representation appropriate for $2 \mathrm{D}$ bilayers; b) Same pattern as in a) but in the representation appropriate for $1 \mathrm{D}$ giant micelles.

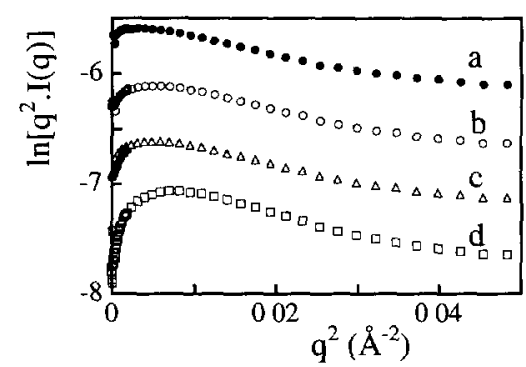

Fig. 4. - Neutron scattering patterns of samples at intermediate salinities in the representation appropriate for $2 \mathrm{D}$ bilayers a) $0293 \mathrm{M} \mathrm{NaCl}$ (precritıcal sample); b) $0.283 \mathrm{M} \mathrm{NaCl}$ (critical sample); c) $0.274 \mathrm{M} \mathrm{NaCl}$; d) $0.260 \mathrm{M} \mathrm{NaCl}$. For clarity, the curves are shifted from one another along the $y$-axis by 0.5 in logarithm units.

representation the pattern appears singular at the origin and diverges at zero $q$.

Figures $5 \mathrm{a}$ and $\mathrm{b}$ show the scattering pattern obtained for the sample with the lowest salinity, i.e., far below the critical condition on the L1 side of it. In Figures 8(a) and (b) the representations appropriate for $2 \mathrm{D}$ bilayers and for $1 \mathrm{D}$ cylinders, respectively, have been used. In a somewhat opposite symmetrical manner, as compared to the highest salinity L3 sample, the pattern has the form of a straight line at low $q$ 's in the $1 \mathrm{D}$ representation (whereas it is singular at zero $q$ in the $2 \mathrm{D}$ representation). This suggests strongly that we have elongated cylindrical micelles at this lower salinity:

$$
I(q) \sim q^{-1} \exp \left(-R_{\mathrm{c}}^{2} q^{2} / 4\right)
$$

The negative slope is then indicative of the average radius of the cylindrical cross-section $R_{\mathrm{c}}$ (slope $=-R_{\mathrm{c}}^{2} / 4$ ) and so we obtain $R_{\mathrm{c}}=15 \AA$, a value which seems realistic for the radius of OBS-pentanol cylindrical micelles.

Thus the morphologies of the primary surfactant structures far from the LMT on the two sides of it are quite unambiguously determined by these patterns: defect-free bilayers at high salinity (L3 side) and long cylindrical micelles at low salinity (L1 side). We now consider the morphological evolution taking place between these two extremes when moving through the critical condition. In Figures $4 \mathrm{a}$ to $4 \mathrm{~d}$ the scattering patterns of the samples at intermediate salinities are represented. For the sake of comparison with defect free membranes, we show 

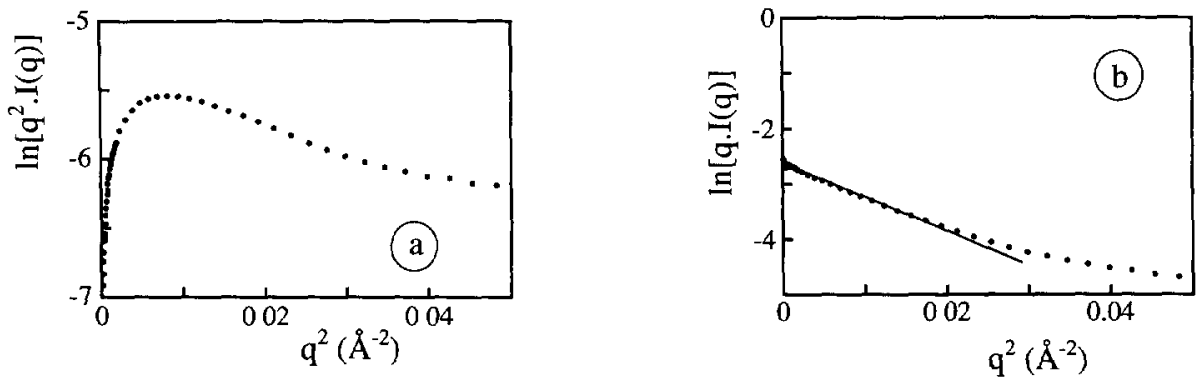

Fig. 5. - a) Neutron scattering pattern of the sample with salinity $0.246 \mathrm{M}$ in the representation appropriate for $2 \mathrm{D}$ bilayers; b) same pattern as in a) but in the representation appropriate for $1 \mathrm{D}$ giant micelles.

these patterns in the appropriate representation for 2D-bilayers. Comparing all patterns, we observe a very progressive evolution. In the high $q$ range, the scattering pattern of the second sample (Fig. 4a) is similar to that of the regular L3 sample at high salinity; but at low $q$ 's a clear downward deviation is seen below the former straight pattern. This suggests that, for this salinity, the flat 2D-bilayer picture remains relevant at small scale (high $q$ 's) but becomes somewhat inadequate at larger scales. Considering successively (Figs. $4 \mathrm{~b}-\mathrm{d}$ ) the patterns at lower and lower salinities, we see that the low $q$ deviations increase progressively. For a while, however, (Figs. 4a-c), the representation for $2 \mathrm{D}$ remains appropriate at high $q$ 's suggesting that, at sufficiently small scales at least, the local morphology is still that of a bilayer. This is no longer the case for the pattern in Figure $4 \mathrm{~d}$ for which we have checked that both representations appropriate for $1 \mathrm{D}$ and $2 \mathrm{D}$ appear equally inadequate (i.e., both are strongly singular at zero $q)$.

\section{Discussion}

The morphologies observed for our very dilute samples at the highest and lowest salinity bilayers and cylinders, respectively - are just the same as those currently found at higher concentrations in the L3 and L1 phases of many ternary systems. However, at higher concentrations, moving from the bilayers in L3 to the long micelles in L1 at constant amphiphile concentration involves at least two macroscopic phase separations: L3 to lamellar and lamellar to L1. In the very dilute range investigated here, the morphological transformation occurs with no macroscopic phase separation, the maximum turbidity standing between the two extreme morphologies being the only remarkable feature observable at the macroscopic scale. At a more microscopic scale, things are even smoother. Looking at the sequence of neutron scattering patterns, we only see a very smooth evolution at local scale: even the "critical sample" at salinity $0.283 \mathrm{M} \mathrm{NaCl}$ (Fig. 4b) shows nothing special within the sequence. This means that the singular behaviour revealed at large scale by the turbidity maximum at the LMT has no counterpart at local scale: this is indeed what is expected at a second-order phase transition. And this observation further supports the interpretation of the the LMT in terms of a critical phenomenon.

Let us first consider the possibility that the LMT corresponds to the second-order symmetry breaking taking place in absence of structural defects for the membrane. On approaching the transition line, the fluctuations of the symmetry order parameter - and therefore those of the mean curvature of the bilayer - increase progressively and diverge at the critical salinity. But, 
as long as we keep the assumption of a defect free membrane, such fluctuations should not affect the structure at scales smaller than the "cell" size of the sponge. Therefore, no change should be seen in the neutron $q$ range for the precritical (Fig. 4a) and critical (Fig. 4b) samples: their scattering patterns should be simply identical to that of the regular L3 sample (Fig. 3a). So the deviations observed for these two samples rather indicate that some "defects" begin to alter the morphology of the membrane even at salinities far above critical (Fig. 4a). These deviations increase monotonically at lower salinities indicating correlatively the formation of more and more defects. A first indication that the "defects" may well be "edges" is suggested by the fact that the morphological evolution ends up in the form of giant cylindrical micelles. The internal structure of a cylindrical micelle is simply identical to that of two edges of opposite signs stuck together side by side. Thus spontaneous formation of edges provides a natural mechanism for a progressive transformation of membranes into giant micelles.

On the other hand, we note that the deviations observed at low $q$ 's bend downwards. This observation further supports the idea that the "defects" are "edges". In order to examine this point, we would like to predict the scattering properties of a membrane with "holes". So we take as geometrical model a flat ideal surface $\Sigma$ of total area $A$, a portion $(A-a)$ of which is occupied by the active matter of the bilayer, the remaining area $(a)$ corresponding to a random distribution of "holes" of typical size $h$. It can be shown along a rather formal algebra that the scattered intensity $I(q)$ of this model behaves asymptotically in the high and low $q$-ranges, respectively, according to:

$$
\begin{gathered}
I\left(q \gg h^{-1}\right)=I_{0}\left(q \gg h^{-1}\right) \\
I\left(q \ll h^{-1}\right)=\left(1-\frac{a}{A}\right) I_{0}\left(q \ll h^{-1}\right)
\end{gathered}
$$

where $I(q)$ is for the membrane with holes and $I_{0}(q)$ is for the defect-free membrane containing the same amount of active matter. An intuitively appealing argument for equations (3) and (4) runs as follows [11]: at large $q$ 's $\left(q \gg h^{-1}\right)$, the scattered intensity measures the total area of membrane per unit volume of sample and is therefore independent of holes. In the same way, at very low $q$ 's, the scattered intensity measures the aggregation number per unit area of the surface $\Sigma$ and is therefore proportional to $(1-a / A)$. Of course, the crossover between the two regimes occurs at $q$ 's of the order of the inverse typical size of the holes $h^{-1}$.

The asymptotic expressions (3) and (4) are actually consistent with the experimental observations. Let us compare the scattering patterns of the precritical and critical samples in Figures $4 \mathrm{a}$ and $\mathrm{b}$ to that of the defect-free L3 sample in Figure 3a. The three patterns are effectively almost identical in the high $q$-range, whereas the downward deviations observed at low $q$ 's for the precritical and critical samples would reveal the finite area fraction $a / A$ of the ideal surface occupied by the holes. Evaluating $a / A$ from the patterns at low $q$ 's in Figures $4 \mathrm{a}$ and $b$, we obtain respectively $15 \%$ for the precritical sample and $30 \%$ for the critical sample. A similar treatment applied to the data at lower salinities would give still higher area fractions of holes (39\% and $60 \%$, respectively, at least for the next two samples); but it is doubtful that the notion of the underlying ideal surface $\Sigma$ could survive at such large area fraction of holes and therefore the evaluation no longer makes sense.

In conclusion, the present neutron scattering data have shown that the critical behaviour at the LMT should not be interpreted only in terms of symmetry breaking in a sponge consisting of a defect free membrane. The scattering patterns rather reveal a noticeable density of structural defects which we presume are "edges" Assuming that at the critical composition, the notion of the underlying ideal orientable surface covered by the bilayer still makes sense, the area fraction of "holes" can be estimated from the scattering pattern: it is at least of the order of $30 \%$. Of 
course, this does not prove that, following the Huse and Leibler scenario, the contour length of the edges diverges at that point: a divergence in size cannot be revealed by measurements that probe the structure at local scale only. But at such area fraction of holes, edges certainly play an important role in the transition mechanism.

\section{References}

[1] Porte G., Marignan J., Bassereau P. and May R, J. Phys. France 49 (1988) 511

[2] Gazeau D, Bellocq A.M, Roux, D. and Zemb T, Europhys. Lett. 9 (1989) 447

[3] Roux D, Coulon C. and Cates M E., J. Phys. Chem 96 (1992) 4174.

[4] Porte G., J Phys. Condens. Matter 4 (1992) 8649.

[5] Coulon C., Roux D. and Bellocq A.M , Phys Rev Lett 66 (1991) 1709.

[6] Roux D., Cates M.E, Olsson U, Ball R.G., Nallet F. and Bellocq A.M., Europhys Lett. 11 (1990) 229.

[7] Vinches C., Coulon C and Roux D., J Phys. II France 4 (1994) 1665.

[8] Huse D. and Leibler S, Phys. Rev. Lett 66 (1991) 437.

[9] Filali M, Porte G., Appell J and Pfeuty P., J Phys. II France 4 (1994) 349.

[10] Bassereau P., Marignan J and Porte G., J Phys France 48 (1987) 673.

[11] We obtained expressions (3) and (4) using the formal development mentioned in the text and the intuitive argument was suggested to us by one of the referees We acknowledge here his contribution. 